\title{
BIOCOMPATIBILITY AND TOLERABILITY OF A PURELY BICARBONATE- BUFFERED PERITONEAL DIALYSIS SOLUTION
}

Lars Weiss, ${ }^{1}$ Bernd Stegmayr, ${ }^{2}$ Gudrun Malmsten, ${ }^{3}$ Mattias Tejde, ${ }^{4}$ Henrik Hadimeri, ${ }^{5}$ Carl E. Siegert, ${ }^{6}$ Jarl Ahlmén, ${ }^{7}$ Rutger Larsson, ${ }^{8}$ Bo Ingman, ${ }^{9}$ Ole Simonsen, ${ }^{10}$ Henk W. van Hamersvelt, ${ }^{11}$ Ann C. Johansson, ${ }^{12}$ Britta Hylander, ${ }^{13}$ Michael Mayr, ${ }^{14}$ Per-Henrik Nilsson, ${ }^{15}$ Per 0 . Andersson, ${ }^{16}$ and Tatiana De los Ríos ${ }^{17}$

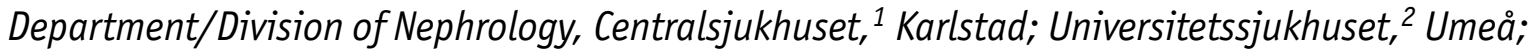
Universitetssjukhuset, ${ }^{3}$ Örebro; Falu Lasarett, ${ }^{4}$ Falun; Höglandsjukhuset Eksjöklinikerna, ${ }^{5}$ Eksjö; Kärnsjukhuset, ${ }^{7}$ Skövde; Universitetssjukhuset, ${ }^{8}$ Linköping; Sunderby Sjukhus, ${ }^{9}$ Luleå; Universitetssjukhuset, ${ }^{10}$ Lund; Department of Nephrology and Transplantation, ${ }^{12}$ Universitetssjukhuset MAS, Malmö; Department/Division of Nephrology, Karolinska Sjukhuset, ${ }^{13}$ Stockholm; Centrallasarettet, ${ }^{15}$ Växjö;

Vrinnevisjukhuset, ${ }^{16}$ Norrköping, Sweden; Department of Nephrology, St. Lucas Andreas Ziekenhuis, ${ }^{6}$ Amsterdam; University Medical Centre Nijmegen, ${ }^{11}$ Nijmegen, The Netherlands; Clinic of Transplantation Immunology and Nephrology, ${ }^{14}$ University Hospital Basel, Basel, Switzerland; Clinical Research Department, ${ }^{17}$ Fresenius Medical Care Deutschland GmbH, Bad Homburg, Germany

$\leftrightarrow$ Background: Novel peritoneal dialysis solutions are characterized by a minimal content of glucose degradation products and a neutral pH. Many studies have shown the biocompatibility of neutral lactate-buffered solutions; however, until now, the effect of purely bicarbonate-buffered solutions has not been intensively studied in vivo.

$\leftrightarrow$ Methods: This study was an open label, prospective, crossover multicenter trial to investigate the biocompatibility of a purely bicarbonate-buffered solution (bicPDF) by measuring biocompatibility parameters such as cancer antigen 125 (CA125) in peritoneal effluent. 55 patients were enrolled in the study. After a 2-week run-in phase, 53 patients could be randomized into 2 groups, starting with either standard lactate-buffered peritoneal dialysis fluid (SPDF) for 12 weeks (phase 1) and then switching to bicPDF for 12 weeks (phase 2), or vice versa. Overnight peritoneal effluents were collected at baseline and at the end of phases 1 and 2 and were tested for CA125, hyaluronic acid, vascular endothelial growth factor (VEGF), tumor necrosis factor-alpha (TNF- $\infty$ ), interleukin 6 (IL-6), interferon gamma (IFN $\gamma$ ), and transforming growth factor-beta ${ }_{1}$ (TGF- 31 ). Total ultrafiltration and residual renal function were also assessed. At the end of the study, pain during fluid exchange and dwell was evaluated using special questionnaires.

$\leftrightarrow$ Results: 34 patients completed the study; 27 of them provided data for analysis of the biocompatibility parameters.

Correspondence to: T. De los Ríos, Clinical Research, Research \& Development, Fresenius Medical Care Deutschland $\mathrm{GmbH}$, Daimlerstrasse 15, 61346 Bad Homburg, Germany.

tatiana.de-los-rios@fmc-ag.com

Received 30 May 2008; accepted 23 February 2009.
CA125 levels in overnight effluent were significantly higher with bicPDF $(61.9 \pm 33.2 \mathrm{U} / \mathrm{L})$ than with SPDF (18.6 \pm $18.2 \mathrm{U} / \mathrm{L}, p<0.001)$. Hyaluronic acid levels were significantly lower after the use of bicPDF $(185.0 \pm 119.6 \mathrm{ng} / \mathrm{mL})$ than after SPDF $(257.4 \pm 174.0 \mathrm{ng} / \mathrm{mL}, p=0.013)$. Both TNF- $\propto$ and TGF- $\beta 1$ showed higher levels with the use of bicPDF than with SPDF. No differences were observed for IL-6, VEGF, or IFN $\gamma$ levels. We observed an improvement in the glomerular filtration rate with the use of bicPDF but no differences were observed for total fluid loss. Pain scores could be analyzed in 23 patients: there was no difference between the solutions.

- Conclusions: The use of a purely bicarbonate-buffered low-glucose degradation product solution significantly changes most of the peritoneal effluent markers measured, suggesting an improvement in peritoneal membrane integrity. Additionally, it seems to have a positive effect on residual renal function.

Perit Dial Int 2009; 29:647-655

www.PDIConnect.com

KEY WORDS: Biocompatibility; cancer antigen 125; residual renal function; peritoneal dialysis fluids; purely bicarbonate buffered.

Tn peritoneal dialysis (PD), the rationale of bicarbon1 ate as buffer substance in the dialysis fluid is evident but requires technological measures to overcome the formation of calcium and magnesium carbonate precipitation (1). This precipitation is avoided by preparing the solution in a double-chamber bag, allowing the use of 
pure bicarbonate as buffer substance. Further, the separation of glucose in one chamber and its sterilization at acidic $\mathrm{pH}$ minimizes the formation of glucose degradation products (GDPs). Mixing the compartments together before use results in a solution at physiological pH (approximately 7.4) with low GDP content (2).

High concentrations of glucose and especially GDPs in conventional PD fluids (PDFs) leads to the formation of advanced glycation end products (AGEs) in the peritoneal membrane (3). Accumulation of AGE in the peritoneum correlates with the development of severe interstitial fibrosis and microvascular sclerosis $(4,5)$. Superior biocompatibility of purely bicarbonate-buffered PDFs compared to conventional PDFs has been demonstrated in several in vitro studies (6-9) and studies in children (10).

The low $\mathrm{pH}$ of PDFs and high $\mathrm{pCO}$, were suggested as pivotal causes of infusion pain $(11,12)$. Henderson et al. (13) found that the intensity of abdominal pain experienced by some patients on continuous ambulatory peritoneal dialysis (CAPD) during infusion of PDF correlates with the storage time of the solutions prior to use. Spectrophotometric analysis of these PDFs revealed a significant presence of GDP in aged solutions. Fewer local effects, including infusion pain during solution exchange, have been observed with the use of biocompatible solutions (e.g., purely bicarbonate-buffered solutions) (14).

Bicarbonate-buffered solutions ( $34 \mathrm{mmol} / \mathrm{L}$ ) have also been shown to be more effective than conventional lactate-buffered solution ( $35 \mathrm{mmol} / \mathrm{L}$ ) in the correction of acidosis (14). Due to their low GDP content and physiological pH, the use of purely bicarbonate-buffered solutions permits a more physiological treatment. This study addresses whether this results in beneficial effects, such as better preservation of the peritoneal membrane and potential clinical effects.

The primary aim of the present study was to investigate the in vivo biocompatibility of a $34 \mathrm{mmol} / \mathrm{L}$ bicarbonate-buffered PDF (bicPDF) in adults. Peritoneal membrane integrity was assessed by several markers, including cancer antigen 125 (CA125), which has been identified to correlate with mesothelial cell mass and turnover (15).

As secondary objectives, the effect of a bicarbonatebuffered PDF on residual renal function (RRF) and its value for pain relief were investigated.

\section{SUBJECTS AND METHODS}

\section{STUDY DESIGN}

This was an open label, prospective, crossover multicenter trial with two treatment periods in random order. A centralized randomization procedure was applied stratifying for diabetes status and time on PD ( $\leq 18$ months and $>18$ months on PD). Stable patients on the stay · safe CAPD system (Fresenius Medical Care, Bad Homburg, Germany) with no PD-related complications during the previous month were asked to sign informed consent. Approval for the study was obtained from the local ethics committees in each country.

All patients underwent an initial 2-week run-in phase with standard lactate-buffered peritoneal dialysis fluid (SPDF) with the appropriate glucose concentration $(1.5 \%, 2.3 \%$, or $4.25 \%)$. Afterward, the patients were randomly assigned to two groups, starting with either SPDF for 12 weeks (phase 1 ) and switching to bicPDF for another 12 weeks (phase 2), or vice versa (Figure 1). All study solutions were manufactured by Fresenius Medical Care, Bad Homburg, Germany, and had compositions as shown in Table 1.

After completing this biocompatibility study phase, pain assessment was performed during a so-called pain assessment phase (Figure 1) to allow blinded administration of the solutions.

\section{BIOCOMPATIBILITY MARKERS IN EFFLUENT AND SERUM}

Effluent samples from overnight dwells of 9 - 12 hours were collected for analysis of biocompatibility markers at the end of the run-in phase (baseline) and at the end of each treatment phase (visits 2, 3, and 4). For the overnight exchanges, the patients used 2-L bags with $2.3 \%$ glucose. Dialysate sampling from the effluent was performed through a 0.2- $\mu$ filter. Samples were stored immediately at $-20^{\circ} \mathrm{C}$ until analysis at a central laboratory (Department of Clinical Research, Medilab Kliniska Laboratorier AB, Täby, Sweden).

Cancer antigen 125 was measured with the Liaison chemiluminescence assay (DiaSorin SpA, Saluggia, Italy). Hyaluronic acid (HA) was determined with a sandwich protein-binding assay in microplate format (Corgenix Ltd., Peterborough, UK). Tumor necrosis factor-alpha (TNF- $\alpha$ ) and interleukin-6 (IL-6) were analyzed with the Quantikine high-sensitivity ELISA (R\&D Systems, Minneapolis, MN, USA). Vascular endothelial growth factor (VEGF), interferon gamma (IFN $\gamma$ ), and transforming growth factor-beta ${ }_{1}$ (TGF- $\beta 1$ ) were measured with the quantitative sandwich enzyme immunoassay technique (R\&D Systems). High-sensitivity C-reactive protein was analyzed with a near-infrared particle immunoassay rate methodology (Synchron LX Systems; Beckman Coulter, Brea, CA, USA). Interleukin- 6 was also analyzed in serum using the same method as used for dialysate samples. All these analyses were performed following the manufacturers' instructions. 


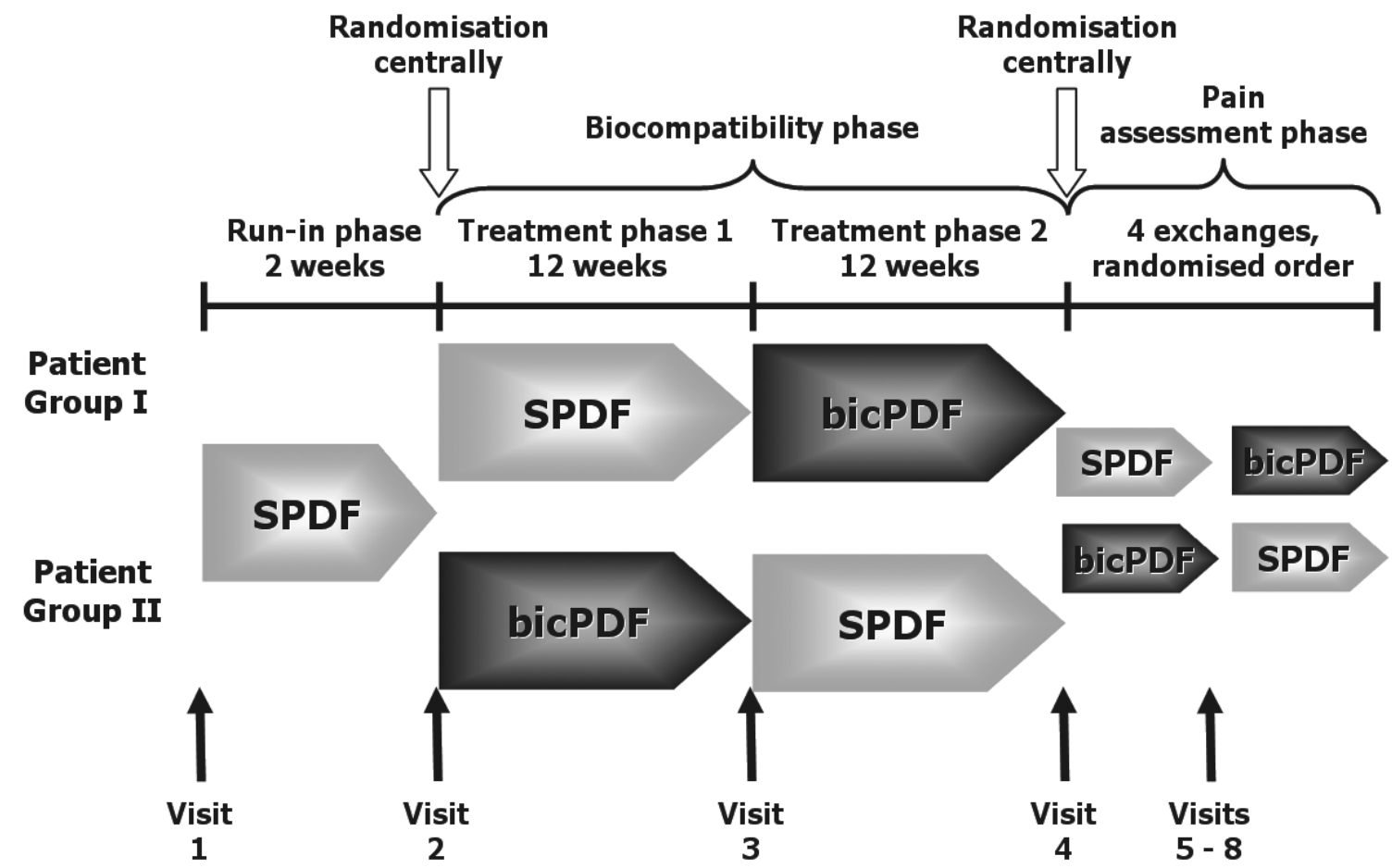

Figure 1 - Diagram of study design. SPDF = standard lactate-buffered peritoneal dialysis fluid; bicPDF = purely bicarbonatebuffered peritoneal dialysis fluid.

TABLE 1

Composition of Study Solutions

\begin{tabular}{lcc}
\hline & SPDF & bicPDF \\
\hline Sodium (mmol/L) & 134 & 134 \\
Calcium (mmol/L) & 1.75 & 1.75 \\
Magnesium (mmol/L) & 0.5 & 0.5 \\
Chloride (mmol/L) & 103.5 & 104.5 \\
L-Lactate (mmol/L) & 35 & - \\
Bicarbonate (mmol/L) & - & 34 \\
Glucose (g/L) & $15.0 / 42.5 / 22.7$ & $15.0 / 42.5 / 22.7$ \\
Osmolarity ${ }^{\text {a }}(\mathrm{m} 0 \mathrm{sm} / \mathrm{L})$ & $358 / 511 / 401$ & $358 / 509 / 399$ \\
pH & approx.5.5 & approx.7.4 \\
\hline
\end{tabular}

SPDF = standard lactate-buffered peritoneal dialysis fluid; bicPDF = purely bicarbonate-buffered peritoneal dialysis fluid. a Theoretical.

\section{RESIDUAL RENAL FUNCTION AND TOTAL FLUID LOSS}

Residual renal function and total fluid loss were assessed during visits 2, 3, and 4. For this purpose, the patients performed a 24-hour dialysate and urine collection, in which both urea and creatinine concentrations were measured. Glomerular filtration rate (GFR) was calculated using the formula GFR $=$ (renal creatinine clearance + renal urea clearance) $\times 0.5$.

Total fluid loss was estimated as the sum of urine volume and net ultrafiltration (UF) volume. For the assess- ment of net UF, the patients weighed the bags before and after each dwell during the day preceding the study visit. The UF of each dwell was calculated as the difference between outflow volume and inflow volume. Net UF volume was then calculated as the sum of the UF of each dwell.

\section{PERITONEAL FUNCTION AND ACID-BASE PARAMETERS}

Peritoneal function and acid-base parameters were measured during visits 2,3 , and 4 . Peritoneal function was assessed using the peritoneal equilibration test according to Twardowski et al. (16).

Acid-base parameters were analyzed by standard methods applied at each study site.

\section{PAIN ASSESSMENT PHASE}

The aim of this phase was to assess pain levels during the exchange and the dwell of the PD solutions. To enable a blinded application to the patients, this phase was added at the end of the otherwise open study. For this purpose, the patients underwent four exchanges at the study center: two with SPDF and two with bicPDF. These exchanges were standardized to $2-\mathrm{L} 4.25 \%$ glucose bags for a 3-hour dwell time. The order in which the patient received the solutions was centrally randomized and independent of the visit. The exchanges were performed 
by the nurse and the bags were blinded; thus, the solution type was unknown to the patients. According to patient preference, these four exchanges were performed either at four different visits or during two visits with two exchanges each.

During these exchanges, the McGill Pain Questionnaire $(12,17)$ and an additional specific questionnaire designed to assess pain at different time points during inflow, dwell, and outflow were applied. The questionnaires comprised a scoring system and had to be read out to the patient at the clinic.

\section{STATISTICAL METHODS}

For the inductive analysis of biocompatibility markers, GFR, and total fluid loss (UF + 24 urine volume), linear mixed models were used and included solution type (bicPDF/SPDF), time (visit 3/visit 4), and the respective baseline values as fixed effects. The individual patient was also implemented as a random effect into these models. For other parameters, descriptive analyses with $95 \%$ confidence intervals for the intraindividual mean differences between the solution types were calculated. If not stated otherwise, results are given as mean \pm standard deviation. The complete analysis was performed with the statistical software SAS 9.1.3 (SAS Institute, (ary, NC, USA).

\section{RESULTS}

\section{STUDY POPULATION}

This study was performed in 13 centers in Sweden, 2 in The Netherlands, and 1 in Switzerland. A total of 55 CAPD patients were recruited (1 center recruited 6 patients, 5 centers recruited 5 patients each, 3 centers recruited 4 patients each, 1 center recruited 3 patients, 3 centers recruited 2 patients each, and 3 centers recruited 1 patient each).

During the run-in phase, 2 patients dropped out of the study (due to patient wish and an adverse event not related to the PD solution); therefore, 53 patients were randomized and started the study phase (Table 2 ). Of these 53, 19 discontinued the treatment prematurely. Reasons for dropout during the treatment with SPDF were transplantation $(n=3)$, patient wish $(n=2)$, peritonitis $(n=1)$, adverse events not related to the PD solution $(n=3)$, and death $(n=2)$. Patients dropped out during the treatment with bicPDF due to patient wish $(n=4)$ and adverse events not related to the PD solution $(n=4)$. Therefore, 34 patients finished the study. In the full analysis set, 27 patients without major pro- tocol violations were included (representing the patients completing the study per protocol). Seven patients could not be included in this analysis set due to missing data for CA125 $(n=3)$, randomization failure $(n=1)$, overnight dwell $<9$ hours $(n=1)$, and implausible data $(n=2)$. In this population, biocompatibility parameters as primary target variables, RRF, total fluid loss, and biochemical parameters were assessed. Twenty-three patients that performed the pain assessment phase and that had complete questionnaires for assessing pain were included in the pain assessment analysis set. The baseline characteristics of all randomized patients and both analysis populations are presented in Table 2.

\section{BIOCOMPATIBILITY MARKERS IN EFFLUENT AND SERUM}

The levels of CA125 measured in the overnight effluents. At baseline it was $17.4 \pm 13.5 \mathrm{U} / \mathrm{L}$. CA125 was significantly higher at the end of the 12-week period with bicPDF $(61.9 \pm 33.2 \mathrm{U} / \mathrm{L})$ than after the period with SPDF $(18.6 \pm 18.2 \mathrm{U} / \mathrm{L}, p<0.001)$ (Figure 2$)$. Hyaluronic acid level in overnight effluent was $244.2 \pm 167.7 \mathrm{ng} / \mathrm{mL}$ at baseline. At the end of the 12-week periods it was significantly lower after bicPDF $(185.0 \pm 119.6 \mathrm{ng} / \mathrm{mL})$ than after SPDF $(257.4 \pm 174.0 \mathrm{ng} / \mathrm{mL}, p=0.013)$ (Figure 2).

Both TNF- $\alpha$ and TGF- $\beta 1$ levels were higher with bicPDF than with SPDF. No significant differences could be demonstrated in overnight effluent levels of IL-6, VEGF, or IFN $\gamma$ with either bicPDF or SPDF. Serum IL- 6 and C-reactive protein levels also did not show significant differences (Table 3).

\section{RESIDUAL RENAL FUNCTION AND TOTAL FLUID LOSS}

At baseline, GFR was $4.48 \pm 3.20 \mathrm{~mL} /$ minute, daily urine volume was $1090 \pm 724 \mathrm{~mL}$, and 24-hour UF was $640 \pm 102 \mathrm{~mL}$.

We determined a GFR of $4.33 \pm 3.42 \mathrm{~mL} /$ minute with SPDF and $4.82 \pm 3.32 \mathrm{~mL} /$ minute $(p=0.011)$ with bicPDF (Figure 3). The daily urine volume was $1398 \pm 975 \mathrm{~mL}$ at the end of the bicPDF phase, which was significantly higher that at the end of the SPDF phase $(1071 \pm 753 \mathrm{~mL}$, $p<0.001)$. At the same time, 24-hour UF tended to be lower after 12 weeks' treatment with bicPDF (494 \pm $771 \mathrm{~mL})$ than with SPDF $(727 \pm 938 \mathrm{~mL}, p=\mathrm{NS})$. No differences in the average glucose strength of the PD solutions between the two phases were observed. The difference in total fluid loss between the two solutions was not significant (Figure 3). No changes in body weight were observed. 
TABLE 2

Baseline Patient Characteristics for All Patients Randomized, the Full Analysis Set (FAS), and the Pain Assessment Analysis Set (PAS) (Values Reported as Mean \pm SD, as Appropriate)

\begin{tabular}{lccc}
\hline & All patients randomized & FAS & PAS \\
\hline$N$ & 53 & 27 & 23 \\
Sex $(\mathrm{M}: \mathrm{F})$ & $31: 22$ & $16: 11$ & $15: 8$ \\
Age $($ years $)$ & $60.9 \pm 14.8$ & $56.0 \pm 16.8$ & $58.7 \pm 16.4$ \\
Height $(\mathrm{cm})$ & $170.9 \pm 9.1$ & $170.3 \pm 10.1$ & $170.3 \pm 9.2$ \\
Weight $(\mathrm{kg})$ & $75.3 \pm 14.9$ & $73.9 \pm 16.8$ & $73.3 \pm 16.2$ \\
Body mass index $\left(\mathrm{kg} / \mathrm{m}^{2}\right)$ & $25.7 \pm 3.9$ & $25.3 \pm 4.0$ & $25.1 \pm 4.1$ \\
Time on peritoneal dialysis (months) & $10.9 \pm 10.8$ & $7.0 \pm 5.4$ & $9.0 \pm 9.2$ \\
Primary renal disease $(n)$ & & & 4 \\
$\quad$ Diabetic nephropathy & 16 & 6 & 8 \\
Glomerulonephritis & 11 & 3 & 3 \\
Hypertensive nephropathy & 9 & 2 & 1 \\
$\quad$ Other vascular disease & 3 & 4 & 3 \\
Polycystic kidney disease & 5 & 3 & 2 \\
Other & 6 & 2 & 2 \\
$\quad$ Unknown & 3 & & 4 \\
\hline
\end{tabular}
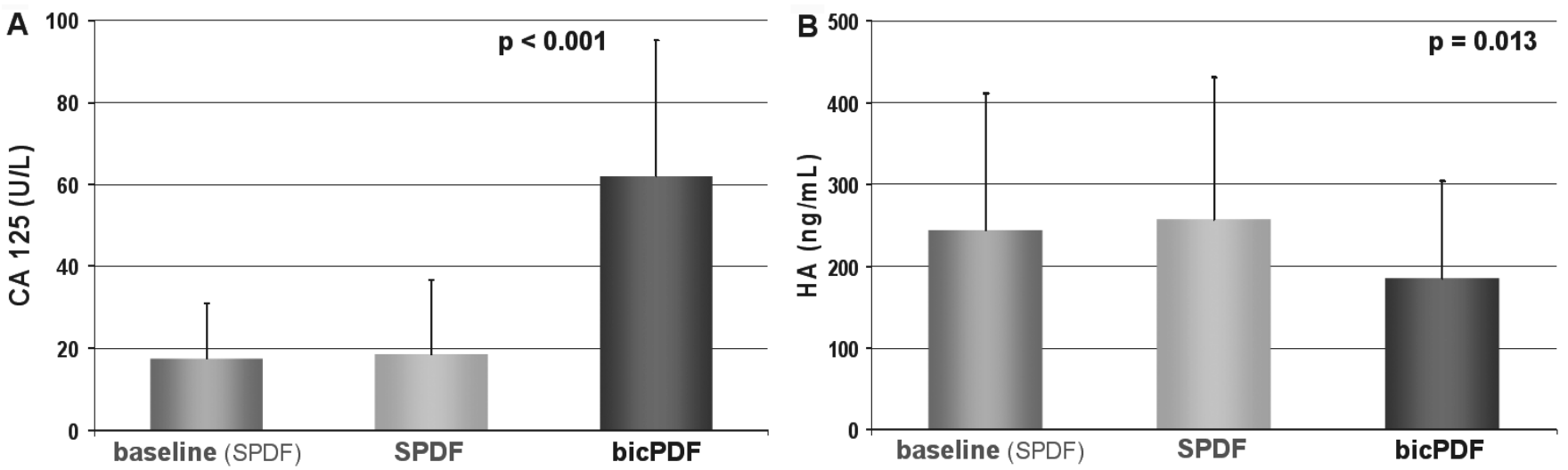

Figure 2 - Calcium antigen 125 (CA125; panel A) and hyaluronic acid (HA; panel B) levels at baseline, at the end of the 12 weeks of treatment with the standard lactate-buffered peritoneal dialysis fluid (SPDF) and the purely bicarbonate-buffered peritoneal dialysis fluid (bicPDF).

TABLE 3

Biocompatibility Markers in Dialysate and Serum (Values Reported as Mean \pm SD)

\begin{tabular}{lcccc}
\hline & Baseline & SPDF & bicPDF & $p$ Value $^{\text {a }}$ \\
\hline IL-6 $(\mathrm{pg} / \mathrm{mL})$ & $23.0 \pm 17.6$ & $21.7 \pm 19.1$ & $25.5 \pm 36.9$ & NS \\
TNF- $\alpha(\mathrm{pg} / \mathrm{mL})$ & $0.8 \pm 0.3$ & $0.6 \pm 0.3$ & $1.0 \pm 0.4$ & $<0.001$ \\
VEGF $(\mathrm{pg} / \mathrm{mL})$ & $47.3 \pm 14.8$ & $40.1 \pm 19.0$ & $44.3 \pm 16.8$ & NS \\
IFN $\gamma(\mathrm{pg} / \mathrm{mL})$ & $1.8 \pm 2.4$ & $2.1 \pm 3.3$ & $1.5 \pm 2.9$ & NS \\
TGF- $\beta 1(\mathrm{pg} / \mathrm{mL})$ & $67.7 \pm 35.3$ & $45.7 \pm 27.1$ & $62.0 \pm 43.8$ & 0.016 \\
IL-6 in serum $(\mathrm{pg} / \mathrm{mL})$ & $5.7 \pm 3.9$ & $4.7 \pm 2.7$ & $5.3 \pm 2.8$ & NS \\
CRP in serum $(\mathrm{mg} / \mathrm{L})$ & $7.0 \pm 13.0$ & $5.4 \pm 9.4$ & $5.2 \pm 9.0$ & NS \\
\hline
\end{tabular}

IL = interleukin; TNF- $\alpha=$ tumor necrosis factor-alpha; VEGF= vascular endothelial growth factor; IFN $\gamma=$ interferon gamma; TGF- $\beta 1=$ transforming growth factor-beta ${ }_{1} ; \mathrm{CRP}=\mathrm{C}$-reactive protein; $\mathrm{SPDF}=$ standard lactate-buffered peritoneal dialysis fluid; bicPDF = purely bicarbonate-buffered peritoneal dialysis fluid.

a $p$ Values result from the linear mixed models analysis. 

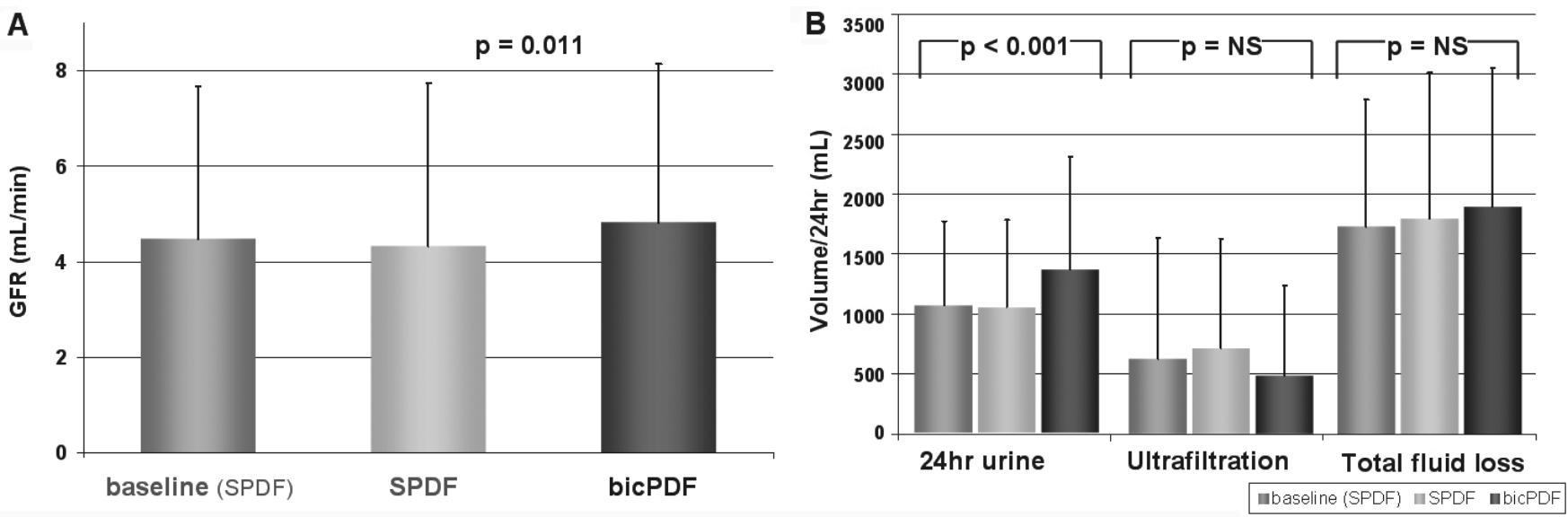

Figure 3-Glomerular filtration rate (GFR; panel A) and 24-hour urine volume, peritoneal net ultrafiltration, and total fluid loss (panel B) with the use of purely bicarbonate-buffered peritoneal dialysis fluid (bicPDF) and standard lactate-buffered peritoneal dialysis fluid (SPDF).

\section{PERITONEAL FUNCTION AND ACID-BASE PARAMETERS}

No changes in dialysate-to-plasma ratios of creatinine and urea were observed with the use of bicPDF and SPDF (data not shown).

$\mathrm{pH}$ and base excess increased with the use of bicPDF compared to SPDF; other acid-base variables were unchanged (Table 4).

\section{PAIN ASSESSMENT PHASE}

On the visual analog scale for pain intensity in the McGill Pain Questionnaire, a value of 0 represents no pain and 10 the most pain possible. The patients marked a pain intensity of $0.29 \pm 0.52$ for bicPDF and $0.54 \pm 1.14$ for SPDF ( $p=$ NS).

In the specific questionnaire on pain related to solution exchange, 4 of 23 patients reported mild or moderate pain with both solutions during inflow. Two patients reported pain during dwells with bicPDF and 3 patients with SPDF. Two patients complained of mild or moder- ate pain during outflow with bicPDF and 5 patients with SPDF.

\section{DISCUSSION}

The present crossover study compared the in vivo biocompatibility of a purely bicarbonate-buffered PDF characterized by physiological $\mathrm{pH}$ and low levels of GDPs against a conventional lactate-buffered PDF. Comparison was made using effluent parameters considered biocompatibility markers of PD solutions, such as CA125 $(15,18-20)$. The concentration of CA125 measured in the overnight effluent was significantly elevated at the end of the bicarbonate treatment phase compared to the conventional PDF treatment phase.

Biocompatibility of lactate- and lactate/bicarbonatebuffered PD solutions with reduced GDP levels and lessacidic or neutral $\mathrm{pH}$ has already been demonstrated in several studies (18-22). Williams et al. (20) observed three- to fourfold increases in CA125 levels in overnight effluent when using the biocompatible lactate-buffered

TABLE 4

Acid-Base Parameters

\begin{tabular}{lcccccc}
\hline & \multicolumn{2}{c}{ Baseline } & \multicolumn{2}{c}{ SPDF } & \multicolumn{2}{c}{ bicPDF } \\
& $N$ & (mean \pm SD) & $N$ & $($ mean \pm SD $)$ & $N$ & $($ mean $\pm S D)$ \\
\hline Bicarbonate $(\mathrm{mmol} / \mathrm{L})$ & 25 & $24.0 \pm 3.3$ & 27 & $24.6 \pm 3.8$ & 27 & $25.4 \pm 3.0$ \\
Venous pH & 27 & $7.3 \pm 0.01$ & 24 & $7.3 \pm 0.1$ & 24 & $7.4 \pm 0.1^{\mathrm{a}}$ \\
Base excess $(\mathrm{mmol} / \mathrm{L})$ & 26 & $-0.7 \pm 3.1$ & 24 & $-1.2 \pm 3.9$ & 27 & $1.0 \pm 3.7^{\mathrm{a}}$ \\
$\mathrm{pCO}_{2}(\mathrm{kPa})$ & 27 & $6.5 \pm 1.0$ & 25 & $6.6 \pm 1.0$ & 25 & $6.5 \pm 1.1$ \\
$\mathrm{pO}_{2}(\mathrm{kPa})$ & 26 & $6.0 \pm 6.0$ & 23 & $5.1 \pm 2.8$ & 22 & $5.0 \pm 2.2$
\end{tabular}

SPDF = standard lactate-buffered peritoneal dialysis fluid; bicPDF = purely bicarbonate-buffered peritoneal dialysis fluid.

a Significant according to $95 \%$ confidence interval of the intraindividual differences between SPDF and bic PDF. 
solution during 3 months. Although it is observed that, with the duration of the PD treatment, CA125 levels decline (21), elevated CA125 related to the use of biocompatible PDF has been observed also in other studies $(19,20,23-25)$ with up to 2 years of treatment (18).

Before the present in vivo study, the effects of purely bicarbonate-buffered solutions on the peritoneal membrane had been investigated only in children (10). The increased CA125 levels in effluent collected during a 4-hour peritoneal equilibration test in children on automated PD (10) and the recently published first results in adults (26) are now confirmed by our study in a larger adult patient population. Increased CA125 levels are considered associated with a better preservation of the mesothelial cell layer, possibly by an increase in mesothelial cell mass and function (27).

In the present study we observed a significant decrease in effluent HA concentrations when patients used bicPDF, suggesting a lower proinflammatory potential of this solution since HA is produced by mesothelial cells and is considered a surrogate marker of peritoneal inflammation (28). Hyaluronic acid is increased during peritonitis (29) and is involved in peritoneal remodeling processes. Our results are in agreement with studies using biocompatible lactate-buffered PD solutions that are also characterized by neutral $\mathrm{pH}$ and low GDP content (18-20).

We also determined the concentrations of IL-6, IFN $\gamma$, TNF- $\alpha$, VEGF, and TGF- $\beta 1$ in overnight dialysate. Whereas no significant differences were observed for IL-6, IFN $\gamma$, or VEGF, both TNF- $\alpha$ and TGF- $\beta 1$ showed a slight but significant increase in the bicPDF phase. These results seem to contradict the hypothesis of a lower proinflammatory effect of biocompatible solutions, as suggested by the reduced $\mathrm{HA}$. However, increased secretion of these mediators could be interpreted as being a result of improved peritoneal macrophage and mesothelial function with the use of bicPDF, which is in line with data from ex vivo and in vitro studies (30-32). As increase CA125 concentration in the peritoneal effluent is considered an indicator of an improved mesothelial cell mass, it might be possible that increased secretion of TNF- $\alpha$ and TGF- $\beta 1$ is a consequence of increased mesothelial cell numbers and improved mesothelial cell function. The relevance of these findings should be interpreted with caution because all levels of TNF- $\alpha$ and TGF- $\beta 1$ in our study were far below the levels found in other studies $(19,20,23)$ and because the levels found with bicPDF were comparable to those at baseline (Table 3 ). An alternative explanation for the increased TNF- $\alpha$ levels during bicPDF might be an increased release of TNF- $\alpha$ by macrophages during peritonitis (33), but the incidence of peritonitis was not different between the two solutions (data not shown).

The purely bicarbonate-buffered PDF induced a significant increase in RRF - measured as residual GFR and diuresis. This improvement in RRF with bicPDF was observed independently of the order in which SPDF and bicPDF were applied, thereby excluding natural decline of renal function as a confounding factor. It is possible that the bicPDF has a positive influence on RRF as such by reducing potential AGE-related systemic damage to the kidney (34). Parallel to the increase in RRF, we observed a slightly but not significantly lower UF volume with bicPDF. This is in accordance with results from previous studies with biocompatible lactate-buffered (20), purely bicarbonate-buffered (35), and mixed lactateand bicarbonate-buffered PDF (36), suggesting beneficial effects not only on the peritoneal membrane but also on RRF. Due to the parallel increase in diuresis and decrease in UF, total fluid elimination was comparable at the end of each study phase. In addition, body weight did not differ at the end of either study phase (74.9 \pm $15.9 \mathrm{~kg}$ with bicPDF vs $75.0 \pm 16.3 \mathrm{~kg}$ with SPDF), thereby excluding differences in fluid balance during the 12-week study periods. However, to draw conclusions, investigations applying precise methods of assessing the effect of conventional and new more biocompatible PD solutions on patients' fluid status are required (37).

One of the other advantages of purely bicarbonatebuffered solutions over lactate-buffered solutions is the capacity to better control metabolic acidosis $(10,14,38)$. In our study, a significant change in acid-base parameters was visible for base excess and $\mathrm{pH}$. Other acid-base parameters were unchanged but were already within normal laboratory ranges at baseline, likely due to the fact that more than $40 \%$ of the patients received oral bicarbonate substitution.

We could not demonstrate a difference in infusion pain between bicPDF and SPDF in our population and hence could not confirm the reduction in infusion pain previously demonstrated for other biocompatible fluids with a (near) neutral pH and low GDP content $(12,14)$. However, the frequency of pain reported by our study population was remarkably low, probably due to the fact that the patients in our study were not selected for the presence of severe infusion pain as in other studies of this subject $(12,14)$.

In conclusion, the use of a purely bicarbonate-buffered PD solution proved to be advantageous for PD patients with increased effluent CA125 concentration. This suggests better preservation of the mesothelial layer. $A$ decrease in HA indicates a decrease in peritoneal inflammation. A better preservation of residual renal function 
might have a possible benefit for patient survival. of note, we also observed small increases in TNF- $\alpha$ and TGF- $\beta 1$ with bicPDF, which seemed to contradict our result with HA. However, these latter results should be interpreted with caution because the levels obtained with bicPDF were comparable to the levels at baseline and because the levels were far below those in other studies with biocompatible peritoneal dialysis fluids. The longterm benefit of treatment with purely bicarbonate-buffered solution should be proven in a well-designed randomized long-term study.

\section{DISCLOSURE}

This study was supported by Fresenius Medical Care.

\section{ACKNOWLEDGMENTS}

We thank all the nurses, monitors, and co-investigators that helped to collect the data, the patients that took part in this study, and the Fresenius Medical Care staff who collaborated with the successful performance of this study.

\section{REFERENCES}

1. Feriani M, Biasioli S, Chiaramonte S, Bragantini L, Brendolan A, Dell'Aquila R, et al. Will bicarbonate-CAPD strengthen the natural defence by having a physiological pH and a natural buffer? Contrib Nephrol 1987; 57:101-9.

2. Tauer A, Knerr T, Niwa T, Schaub TP, Lage C, PasslickDeetjen $\mathrm{J}$, et al.In vitro formation of $\mathrm{N}$ (epsilon)-(carboxymethyl) lysine and imidazolones under conditions similar to continuous ambulatory peritoneal dialysis. Biochem Biophys Res Commun 2001; 280:1408-14.

3. Mortier S, Faict D, Lameire NH, De Vriese AS. Benefits of switching from a conventional to a low-GDP bicarbonate/ lactate-buffered dialysis solution in a rat model. Kidney Int 2005; 67:1559-65.

4. Honda K, Nitta K, Horita S, Yumura W, Nihei H, Nagai R, et al. Accumulation of advanced glycation end products in the peritoneal vasculature of continuous ambulatory peritoneal dialysis patients with low ultra-filtration. Nephrol Dial Transplant 1999; 14:1541-9.

5. Nakayama M, Kawaguchi Y, Yamada K, Hasegawa T, Takazoe $\mathrm{K}$, Katoh $\mathrm{N}$, et al. Immunohistochemical detection of advanced glycosylation end-products in the peritoneum and its possible pathophysiological role in CAPD. Kidney Int 1997; 51:182-6.

6. Topley N, Kaur D, Petersen MM, Jorres A, Passlick-Deetjen $\mathrm{J}$, Coles GA, et al. Biocompatibility of bicarbonate buffered peritoneal dialysis fluids: influence on mesothelial cell and neutrophil function. Kidney Int 1996; 49:1447-56.

7. Jorres A, Bender TO, Finn A, Witowski J, Frohlich S, Gahl $G M$, et al. Biocompatibility and buffers: effect of bicar- bonate-buffered peritoneal dialysis fluids on peritoneal cell function. Kidney Int 1998; 54:2184-93.

8. Plum J, Razeghi P, Lordnejad RM, Perniok A, Fleisch M, Fussholler $A$, et al. Peritoneal dialysis fluids with a physiologic $\mathrm{pH}$ based on either lactate or bicarbonate buffereffects on human mesothelial cells. Am J Kidney Dis 2001; 38:867-75.

9. Mortier S, De Vriese AS, McLoughlin RM, Topley N, Schaub TP, Passlick-Deetjen J, et al. Effects of conventional and new peritoneal dialysis fluids on leukocyte recruitment in the rat peritoneal membrane. J Am Soc Nephrol 2003; 14:1296-306.

10. Haas S, Schmitt CP, Arbeiter K, Bonzel KE, Fischbach M, John $U$, et al. Improved acidosis correction and recovery of mesothelial cell mass with neutral-pH bicarbonate dialysis solution among children undergoing automated peritoneal dialysis. J Am Soc Nephrol 2003; 14:2632-8.

11. Cancarini GC, Faict D, De Vos C, Guiberteau R, Tranaeus A, Minetti $L$, et al. Clinical evaluation of a peritoneal dialysis solution with $33 \mathrm{mmol} / \mathrm{L}$ bicarbonate. Perit Dial Int 1998; 18:576-82.

12. Mactier RA, Sprosen TS, Gokal R, Williams PF, Lindbergh $M$, Naik RB, et al. Bicarbonate and bicarbonate/lactate peritoneal dialysis solutions for the treatment of infusion pain. Kidney Int 1998; 53:1061-7.

13. Henderson IS, Couper IA, Lumsden AB. Potentially irritant glucose metabolites in unused CAPD fluid. In: Maher JF, Winchester JF, eds. Frontiers in Peritoneal Dialysis. New York: Field, Rich and Associates; 1986.

14. Feriani M, Kirchgessner J, La Greca G, Passlick-Deetjen J. Randomized long-term evaluation of bicarbonate-buffered CAPD solution. Kidney Int 1998; 54:1731-8.

15. Visser CE, Brouwer-Steenbergen JJ, Betjes MG, Koomen GC, Beelen RH, Krediet RT. Cancer antigen 125: a bulk marker for the mesothelial mass in stable peritoneal dialysis patients. Nephrol Dial Transplant 1995; 10:64-9.

16. Twardowski ZJ, Nolph KD, Khanna R, Prowant BF, Ryan LP, Moore HL, et al. Peritoneal equilibration test. Perit Dial Bull 1987; 7:138-47.

17. Burckhardt CS, Bjelle A. A Swedish version of the shortform McGill Pain Questionnaire. Scand J Rheumatol 1994; 23:77-81.

18. Rippe B, Simonsen 0 , Heimburger 0 , Christensson A, Haraldsson B, Stelin G, et al. Long-term clinical effects of a peritoneal dialysis fluid with less glucose degradation products. Kidney Int 2001; 59:348-57.

19. Jones S, Holmes CJ, Krediet RT, Mackenzie R, Faict D, Tranaeus A, et al. Bicarbonate/lactate-based peritoneal dialysis solution increases cancer antigen 125 and decreases hyaluronic acid levels. Kidney Int 2001; 59: 1529-38.

20. Williams JD, Topley N, Craig KJ, Mackenzie RK, Pischetsrieder M, Lage C, et al. The Euro-Balance Trial: the effect of a new biocompatible peritoneal dialysis fluid (balance) on the peritoneal membrane. Kidney Int 2004; 66:408-18. 
21. Bouts AH, GroothoffJW, van Amstel SP, Zweers MM, Davin JC, Krediet RT. Dialysate cancer antigen 125 levels in children treated with peritoneal dialysis. Adv Perit Dial 2000; 16:328-31.

22. Krediet RT. Dialysate cancer antigen 125 concentration as marker of peritoneal membrane status in patients treated with chronic peritoneal dialysis. Perit Dial Int 2001; 21:560-7.

23. Fusshoeller A, Plail M, Grabensee B, Plum J. Biocompatibility pattern of a bicarbonate/lactate-buffered peritoneal dialysis fluid in APD: a prospective, randomized study. Nephrol Dial Transplant 2004; 19:2101-6.

24. Ho-dac-Pannekeet MM, Hiralall JK, Struijk DG, Krediet RT. Longitudinal follow-up of CA125 in peritoneal effluent. Kidney Int 1997; 51:888-93.

25. Szeto CC, Chow KM, Lam CW, Leung CB, Kwan BC, Chung $\mathrm{KY}$, et al. Clinical biocompatibility of a neutral peritoneal dialysis solution with minimal glucose-degradation products-a 1-year randomized control trial. Nephrol Dial Transplant 2007; 22:552-9.

26. Theodoridis M, Passadakis P, Kriki P, Gioka T, Panagoutsos $S$, Mourvati $E$, et al. The alteration of dialysate cancer antigen 125 concentration under a biocompatible bicarbonate peritoneal dialysis solution and the preservation of the mesothelial cell viability. Ren Fail 2008; 30:161-7.

27. Hömme M, Hackert T, Schäfer J, Schaefer F, Schmitt CP. Peritoneal dialysis solutions acutely regulate CA125 expression in human primary mesothelial cells [Abstract]. J Am Soc Nephrol 2007; 18:74A.

28. Yung S, Chan TM. Hyaluronan-regulator and initiator of peritoneal inflammation and remodeling. Int $\mathrm{J}$ Artif Organs 2007; 30:477-83.

29. Yung S, Coles GA, Williams JD, Davies M. The source and possible significance of hyaluronan in the peritoneal cavity. Kidney Int 1994; 46:527-33.

30. Mackenzie RK, Holmes CJ, Moseley A, Jenkins JP, Williams $\mathrm{JD}$, Coles GA, et al. Bicarbonate/lactate- and bicarbonate-buffered peritoneal dialysis fluids improve ex vivo peritoneal macrophage TNFalpha secretion. J Am Soc Nephrol 1998; 9:1499-506.

31. Martikainen TA, Teppo AM, Gronhagen-Riska C, Ekstrand AV. Glucose-free dialysis solutions: inductors of inflammation or preservers of peritoneal membrane? Perit Dial Int 2005; 25:453-60.

32. Liberek T, Lichodziejewska-Niemierko M, KnopinskaPosluszny W, Schaub TP, Kirchgessner J, Passlick-Deetjen $\mathrm{J}$, et al. Generation of TNFalpha and interleukin- 6 by peritoneal macrophages after overnight dwells with bicarbonate- or lactate-buffered dialysis fluid. Perit Dial Int 2002; 22:663-9.

33. Fieren MW, van den Bemd GJ, Bonta IL, Ben Efraim S. Peritoneal macrophages from patients on continuous ambulatory peritoneal dialysis have an increased capability to release tumour necrosis factor during peritonitis. J Clin Lab Immunol 1991; 34:1-9.

34. Schmitt CP, von Heyl D, Rieger S, Arbeiter K, Bonzel KE, Fischbach $M$, et al. Reduced systemic advanced glycation end products in children receiving peritoneal dialysis with low glucose degradation product content. Nephrol Dial Transplant 2007; 22:2038-44.

35. Montenegro J, Saracho RM, Martinez IM, Munoz RI, Ocharan JJ, Valladares E. Long-term clinical experience with pure bicarbonate peritoneal dialysis solutions. Perit Dial Int 2006; 26:89-94.

36. Fang W, Mullan R, Shah H, Mujais S, Bargman JM, Oreopoulos DG. Comparison between bicarbonate/lactate and standard lactate dialysis solution in peritoneal transport and ultrafiltration: a prospective, crossover singledwell study. Perit Dial Int 2008; 28:35-43.

37. Konings CJ, Kooman JP, van der Sande FM, Leunissen KM. Fluid status in peritoneal dialysis: what's new? Perit Dial Int 2003; 23:284-90.

38. Feriani M, Passlick-Deetjen J, Jaeckle-Meyer I, La Greca $\mathrm{G}$. Individualized bicarbonate concentrations in the peritoneal dialysis fluid to optimize acid-base status in CAPD patients. Nephrol Dial Transplant 2004; 19:195-202. 